\section{Influência das características hospitalares na realização de cesárea eletiva na Região Sudeste do Brasil}

\author{
Influence of hospital characteristics on the \\ performance of elective cesareans in \\ Southeast Brazil
}

Laura Zaiden 1,2

Marcos Nakamura-Pereira 1

Maria Auxiliadora Mendes Gomes 1

Ana Paula Esteves-Pereira 3

Maria do Carmo Leal 3

doi: 10.1590/0102-311X00218218

\title{
Resumo
}

Este artigo tem como objetivo avaliar a influência das características hospitalares sobre a chance de realização de cesariana eletiva na Região Sudeste do Brasil. Foram utilizados dados da pesquisa Nascer no Brasil, realizada entre fevereiro de 2011 e outubro de 2012. A presente análise inclui a amostra da Região Sudeste, compreendendo 10.155 mulheres. O grupo de mulheres submetidas à cesariana eletiva foi comparado ao de mulheres que entraram em trabalho de parto ou foram submetidas à indução do parto, independentemente se fizeram cesariana intraparto ou parto vaginal. Com exceção da idade gestacional, todas as características obstétricas analisadas mostraram-se associadas à cesariana eletiva. Nesse grupo, 60,5\% não possuíam cesariana prévia à gestação atual e 64,7\% eram de baixo risco. Dentre os partos com financiamento público, observou-se maior chance de cesárea eletiva nas mulheres que foram atendidas nos hospitais com $<1.500$ $(O R=2,11$; IC95\%: 1,37-3,26) e entre 1.500-2.999 partos/ano $(O R=1,45$; IC95\%: 1,04-2,02) e nos hospitais mistos (OR = 1,81; IC95\%: 1,37-2,39). Nos hospitais mistos, a magnitude da associação é maior quando localizados em não capitais com volume > 3.000 partos/ano (OR = 3,45; IC95\%: 1,68$7,08)$ e atinge seu maior valor nos hospitais localizados em não capitais com volume < 3.000 partos/ano (OR = 4,08; IC95\%: 2,61-6,37). Em contrapartida, não observou-se associação entre cesariana eletiva e os hospitais públicos localizados em não capitais do Sudeste. As prevalências de cesariana eletiva nos hospitais públicos da Região Sudeste são altas quando comparadas a outros países, e sofrem importante influência das características hospitalares.

Cesárea; Parto Normal; Sistema Único de Saúde; Hospitais Públicos;

Trabalho de Parto Induzido

Correspondência

M. Nakamura-Pereira

Instituto Nacional de Saúde da Mulher, da Criança e do Adolescente Fernandes Figueira, Fundação Oswaldo Cruz. Av. Rui Barbosa 716, 30 andar, Rio de Janeiro, $R J$ 22250-020, Brasil.

marcosnakamura@globo.com

${ }^{1}$ Instituto Nacional de Saúde da Mulher, da Criança e do Adolescente Fernandes Figueira, Fundação Oswaldo Cruz, Rio de Janeiro, Brasil.

2 Faculdade de Medicina de Petrópolis, Petrópolis, Brasil. ${ }^{3}$ Escola Nacional de Saúde Pública Sergio Arouca, Fundação Oswaldo Cruz, Rio de Janeiro, Brasil. 


\section{Introdução}

O Brasil é conhecido no cenário mundial pela sua alta prevalência de cesarianas. Nas últimas duas décadas, houve um aumento importante na frequência dessa cirurgia, atingindo $57 \%$ de todos os nascimentos em 2014 (Sistema de Informações sobre Nascidos Vivos. http://tabnet.datasus.gov.br/ cgi/tabcgi.exe?sinasc/cnv/nvuf.def, acessado em 16/Mai/2019). Recentes análises não identificaram benefícios, no nível populacional, de prevalências de cesárea acima de 15\% 1,2. A cesariana, quando clinicamente indicada, salva mães e bebês, entretanto, seu uso indiscriminado pode aumentar o risco de complicações para ambos 3,4,5,6, tornando importante conhecer os prováveis motivos para prevalências tão altas.

A distribuição das prevalências de cesarianas é heterogênea no país, sendo maiores nas regiões mais ricas (Sudeste, Sul e Centro-oeste), em mulheres com maior poder aquisitivo, mais velhas e com mais anos de escolaridade 7. Quando se considera o tipo de financiamento, há uma grande diferença nas prevalências de cesariana, que atinge $89 \%$ dos partos com financiamento privado e $43 \%$ dos com financiamento público ${ }^{8}$. Além disso, existem no Brasil três tipos de instituições hospitalares quanto ao financiamento: os hospitais estritamente públicos, financiados pelo governo; os hospitais estritamente privados; e os hospitais mistos, que são instituições privadas que podem receber tanto financiamento público quanto privado. Esses últimos estabelecimentos podem eventualmente atender somente a pacientes com financiamento público, mas comumente atendem tanto a pacientes particulares quanto aos do sistema público. Em 2009, de acordo com o Cadastro Nacional dos Estabelecimentos de Saúde (CNES), dos 7.161 hospitais cadastrados, 40,6\% eram públicos, 9,8\% privados e 46,6\% mistos, sendo que a maior concentração destes últimos foi encontrada na Região Sudeste 9.

Já é conhecido que no setor privado a frequência de cesarianas é alta em todo o país, entretanto, na assistência com financiamento do Sistema Único de Saúde (SUS) estes números podem sofrer variações importantes de acordo com o tamanho do hospital, o tipo de hospital (público ou misto) e, provavelmente por sua localização, se nas capitais ou no interior dos estados 10. Algumas pesquisas já evidenciaram associação entre características hospitalares e as prevalências de cesariana 10,11,12,13. A análise dessas características é importante para o entendimento da sua participação no aumento da realização dessa cirurgia, a fim de se formular propostas para a sua redução.

Portanto, o presente estudo tem como objetivo avaliar a influência das características hospitalares sobre o risco de cesarianas eletivas na Região Sudeste do país.

\section{Metodologia}

O estudo Nascer no Brasil é um inquérito nacional sobre parto e nascimento, de base hospitalar, realizado no período de fevereiro de 2011 a outubro de 2012. A amostra foi composta por puérperas e seus recém-nascidos, com o objetivo de avaliar as condições de assistência ao parto e nascimento no país. Foram obtidas amostras de 266 hospitais, nos quais entrevistaram-se 90 puérperas em cada um deles. Os critérios de elegibilidade foram hospitais que realizaram mais de 500 partos no ano de 2007 e nos quais ocorreram 78,6\% de todos os nascimentos do Brasil neste mesmo ano 14.

A amostra foi selecionada em três estágios. No primeiro, os hospitais foram estratificados pelas cinco macrorregiões do país, localização (capital ou não) e por tipo de hospital (privado, público ou misto). No segundo estágio, um método de amostragem inversa foi realizado para selecionar o número de dias (mínimo de sete) necessários para entrevistar 90 puérperas em cada hospital. No terceiro estágio, todas as mulheres que tiveram parto hospitalar de um nascido vivo, independentemente do peso e da idade gestacional, ou um natimorto com mais de $500 \mathrm{~g}$ e/ou idade gestacional maior que 22 semanas foram convidadas a participar. Os pesos amostrais foram estabelecidos pelo inverso da probabilidade da inclusão de cada puérpera na amostra. Um processo de calibração foi usado para garantir que as estimativas totais fossem equivalentes ao número de nascimentos em hospitais com 500 ou mais partos/ano em 2011. Essa calibração foi necessária, pois a seleção dos hospitais foi realizada com base nos dados do Sistema de Informações sobre Nascidos Vivos (SINASC) de 2007. Informações detalhadas sobre a coleta de dados e o desenho da amostra podem ser encontradas em outros artigos 14,15 . 
Este estudo incluiu todas as puérperas amostradas na Região Sudeste, totalizando 10.155 dentre as 23.894 puérperas entrevistadas do Nascer no Brasil. A amostra foi dividida em dois grupos para fins de comparação: mulheres submetidas à cesariana eletiva e mulheres submetidas à cesariana intraparto e parto vaginal. Para a definição dessas variáveis foram usadas informações sobre o início do trabalho de parto (espontâneo, induzido ou cesariana antes do trabalho de parto). Considerou-se como cesariana intraparto as realizadas durante o trabalho de parto ou após o início da indução do mesmo. Como cesariana eletiva considerou-se a cirurgia realizada antes do início do trabalho de parto e que não tiveram o trabalho de parto induzido. Foram consideradas como tendo trabalho de parto aquelas que atingiram $4 \mathrm{~cm}$ ou mais de dilatação (fase ativa do trabalho de parto). As mulheres com menos de $4 \mathrm{~cm}$ de dilatação que eventualmente estivessem na fase latente do trabalho de parto e que foram submetidas à cesariana foram incluídas no grupo da cesariana eletiva.

As características hospitalares investigadas foram: tipo de hospital (público, misto, privado); fonte pagadora do parto (pública ou privada); localização do hospital (capital ou não); número de partos/ ano (<1.500, 1.500-2.999, $\geq 3.000$ ); nível de complexidade do hospital (ausência de leitos de unidade intermediária neonatal [UI] e unidade de terapia intensiva [UTI] neonatal, presença apenas de leitos de UI neonatal, presença de leitos de UI e UTI neonatal). Foi definido como fonte pagadora pública as mulheres que tiveram parto em hospitais públicos ou em hospitais mistos com pagamento financiado pelo SUS, e como fonte pagadora privada as mulheres que tiveram partos financiados por plano de saúde ou por desembolso direto, sejam em hospitais privados ou mistos.

Também foram incluídas variáveis relativas às características socioeconômicas e obstétricas: idade (< 20, 20-34, > 35 anos); situação conjugal (morando com parceiro ou não); anos de escolaridade ( $\leq 7$, 8-10, $\geq 11$ ); cesariana anterior (sim, não); tipo de gestação (única, múltipla); apresentação fetal (cefálica, não cefálica); gestação de alto risco (sim, não); obesidade (IMC $\geq 30 \mathrm{~kg} / \mathrm{m}^{2}$ ou não); macrossomia (peso ao nascer $\geq 4.000 \mathrm{~g}$ ou não) e idade gestacional ( $<37 \mathrm{ou} \geq 37$ semanas). Definiu-se como gestação de alto risco mulheres que apresentavam uma ou mais das seguintes condições: hipertensão gestacional/ pré-eclâmpsia, hipertensão crônica, eclâmpsia, diabetes pré-gestacional, diabetes gestacional, doenças crônicas graves, infecção no momento da internação para o parto (incluindo infecção do trato urinário e outra infecção grave, como corioamnionite e pneumonia), descolamento prematuro da placenta, placenta prévia, crescimento intrauterino restrito e malformações fetais. Essas variáveis foram usadas no estudo para controle de confundimento.

Todos os dados foram coletados dos prontuários médicos das mulheres e dos recém-nascidos, exceto os dados das características socioeconômicas que foram coletados por meio de entrevista face a face com as puérperas. As características hospitalares foram coletadas em entrevistas com os diretores dos hospitais.

A análise compreendeu a distribuição de frequência relativa das variáveis estudadas de acordo com o tipo de parto: cesariana eletiva e cesariana intraparto/parto vaginal e, em uma segunda análise, foi realizada a estratificação por fonte pagadora pública e privada. Posteriormente, utilizando-se apenas os dados pertencentes ao financiamento público, foi realizada análise pelo teste qui-quadrado e por modelos de regressão logística múltipla para analisar as variáveis associadas ao desfecho. O primeiro modelo foi ajustado por todas as variáveis socioeconômicas e obstétricas associadas à cesárea eletiva $(\mathrm{p}<0,05)$ simultaneamente, a fim de avaliar a relação das características hospitalares com o desfecho, independentemente entre si. No segundo modelo, igualmente de forma simultânea, a razão de chance foi ajustada também pelas variáveis hospitalares, para avaliar a diferença das razões de chances para cesárea eletiva destas variáveis, uma vez que as demais características hospitalares entraram no modelo.

Foi realizada uma categorização dos hospitais de acordo com a localização (capital ou não), o número de partos/ano $(<3.000,>3.000)$ e o tipo de hospital (SUS, misto, privado), com posterior análise por regressão logística, ajustando-se para as características obstétricas e socioeconômicas. Os hospitais privados não foram categorizados quanto às demais características devido à alta prevalência de cesarianas em todos os cenários.

As variáveis que apresentaram valor de $\mathrm{p}<0,05$ foram identificadas como fatores associados independentemente à cesariana eletiva, sendo calculadas as odds ratios (OR) ajustadas e seus respectivos intervalos de 95\% de confiança (IC95\%). 
Este estudo seguiu as orientações do Conselho Nacional de Saúde, que fornece diretrizes e padrões de pesquisa em seres humanos, e foi aprovado pelo Comitê de Ética em Pesquisa da Escola Nacional de Saúde Pública Sergio Arouca, Fundação Oswaldo Cruz (CEP/ENSP no 92/2010). Os cuidados necessários foram tomados para garantir a privacidade e a confidencialidade das informações. Foi obtida aprovação de todas as juntas de revisão institucional de cada um dos 266 hospitais participantes. Todos os diretores dos hospitais e todas as puérperas assinaram o consentimento informado.

\section{Resultados}

Com exceção da idade gestacional, todas as características obstétricas mostraram-se associadas à cesariana eletiva. Entre as pacientes submetidas à cesariana eletiva, 14,8\% tinham mais de 34 anos de idade e $65 \%$ possuíam escolaridade $\geq 11$ anos. Com relação às características obstétricas, 60,5\% das mulheres não possuíam cesariana prévia à gestação atual, 64,7\% eram de risco habitual obstétrico, 93,4\% dos fetos estavam cefálicos, 14,1\% eram obesas e 4,9\% dos fetos eram macrossômicos (Tabela 1). Quanto às características hospitalares, observa-se que as mulheres atendidas em hospitais privados, fora da capital e em hospitais com < 1.500 partos/ano tiveram maior prevalência de realização de cesariana eletiva (Tabela 1). Em relação ao nível de complexidade do hospital não houve diferença significativamente estatística entre o tipo de parto e a presença ou não de leitos em unidade intermediária e/ou de terapia intensiva neonatal.

Tabela 1

Características hospitalares, socioeconômicas e obstétricas das mulheres de acordo com o tipo de parto (cesariana eletiva e cesariana intraparto/ parto vaginal).

\begin{tabular}{|c|c|c|c|c|c|}
\hline \multirow[t]{3}{*}{ Características } & \multirow{3}{*}{$\begin{array}{l}\text { Total } \\
\text { n (\%) }\end{array}$} & \multicolumn{2}{|c|}{ Tipo de parto } & \multirow{2}{*}{$\begin{array}{c}\text { Prevalência } \\
\text { de cesariana } \\
\text { eletiva }\end{array}$} & \multirow[t]{3}{*}{ Valor de $p$} \\
\hline & & $\begin{array}{l}\text { Cesariana } \\
\text { eletiva }\end{array}$ & $\begin{array}{l}\text { Cesariana } \\
\text { intraparto ou } \\
\text { parto vaginal }\end{array}$ & & \\
\hline & & n (\%) & n (\%) & $\%$ & \\
\hline \multicolumn{6}{|l|}{ Tipo de hospital } \\
\hline Público & $3.453(34,0)$ & $978(21,1)$ & $2.475(44,9)$ & 28,3 & $<0,001$ \\
\hline Misto & $5.127(50,5)$ & $2.350(50,7)$ & $2.777(50,3)$ & 45,8 & \\
\hline Privado & $1.575(15,5)$ & $1.311(28,3)$ & $264(4,8)$ & 83,2 & \\
\hline \multicolumn{6}{|l|}{ Localização do hospital } \\
\hline Capital & $3.429(33,8)$ & $1.321(28,5)$ & $2.108(38,2)$ & 38,5 & 0,04 \\
\hline Não capital & $6.726(66,2)$ & $3.318(71,5)$ & $3.408(61,8)$ & 49,3 & \\
\hline \multicolumn{6}{|l|}{ Número de partos/ano } \\
\hline$<1.500$ & $3.045(30,0)$ & $1.739(37,5)$ & $1.306(23,7)$ & 57,1 & $<0,001$ \\
\hline $1.500-2.999$ & $2.649(26,1)$ & $1.307(28,2)$ & $1.342(24,3)$ & 49,3 & \\
\hline$\geq 3.000$ & $4.461(43,9)$ & $1.593(34,3)$ & $2.868(52,0)$ & 35,7 & \\
\hline \multicolumn{6}{|l|}{ Complexidade do hospital } \\
\hline Sem leitos de UI e UTI neonatal & $1.604(15,8)$ & $755(16,3)$ & $849(15,4)$ & 47,0 & 0,95 \\
\hline Com leitos de UI neonatal, sem UTI neonatal & $1.279(12,6)$ & $578(12,4)$ & $701(12,7)$ & 45,1 & \\
\hline Tem leitos de UTI neonatal & $7.272(71,6)$ & $3.306(71,3)$ & $3.966(71,9)$ & 45,4 & \\
\hline \multicolumn{6}{|l|}{ Idade (anos) } \\
\hline$\leq 19$ & $1.686(16,6)$ & $449(9,7)$ & $1.237(22,4)$ & 26,6 & $<0,001$ \\
\hline $20-34$ & $7.342(72,3)$ & $3.500(75,5)$ & $3.842(69,7)$ & 47,6 & \\
\hline$\geq 35$ & $1.127(11,1)$ & $690(14,8)$ & $437(7,9)$ & 61,2 & \\
\hline
\end{tabular}

(continua) 
Tabela 1 (continuação)

\begin{tabular}{|c|c|c|c|c|c|}
\hline \multirow[t]{3}{*}{ Características } & \multirow[t]{2}{*}{ Total } & \multicolumn{2}{|c|}{ Tipo de parto } & \multirow{2}{*}{$\begin{array}{c}\text { Prevalência } \\
\text { de cesariana } \\
\text { eletiva }\end{array}$} & \multirow[t]{3}{*}{ Valor de $p$} \\
\hline & & $\begin{array}{l}\text { Cesariana } \\
\text { eletiva }\end{array}$ & $\begin{array}{c}\text { Cesariana } \\
\text { intraparto ou } \\
\text { parto vaginal }\end{array}$ & & \\
\hline & n (\%) & n (\%) & n (\%) & $\%$ & \\
\hline \multicolumn{6}{|l|}{ Anos de escolaridade } \\
\hline$\leq 7$ & $2.023(20,0)$ & $686(14,8)$ & $1.337(24,3)$ & 33,9 & $<0,001$ \\
\hline $8-10$ & $2.637(26,1)$ & $930(20,1)$ & $1.707(31,1)$ & 35,2 & \\
\hline$\geq 11$ & $5.456(53,9)$ & $3.006(65,0)$ & $2.450(44,6)$ & 55,0 & \\
\hline \multicolumn{6}{|l|}{ Situação conjugal } \\
\hline Não mora com o parceiro & $2.185(21,5)$ & $806(17,4)$ & $1.379(25,0)$ & 36,8 & $<0,001$ \\
\hline Mora com o parceiro & $7.964(78,5)$ & $3.831(82,6)$ & $4.133(75,0)$ & 48,1 & \\
\hline \multicolumn{6}{|l|}{ Cesariana prévia } \\
\hline Não & $7.825(77,1)$ & $2.805(60,5)$ & $5.020(91,0)$ & 35,8 & $<0,001$ \\
\hline Sim & $2.330(22,9)$ & $1.834(39,5)$ & $496(9,0)$ & 78,7 & \\
\hline \multicolumn{6}{|l|}{ Tipo de gestação } \\
\hline Única & $10.055(99,0)$ & $4.571(98,5)$ & $5.484(99,4)$ & 45,4 & 0,03 \\
\hline Múltipla & $100(1,0)$ & $68(1,5)$ & $32(0,6)$ & 68,0 & \\
\hline \multicolumn{6}{|l|}{ Apresentação fetal } \\
\hline Cefálica & $9.758(96,1)$ & $4.331(93,4)$ & $5.427(98,4)$ & 44,3 & $<0,001$ \\
\hline Não cefálica & $397(3,9)$ & $308(6,6)$ & $89(1,6)$ & 77,5 & \\
\hline \multicolumn{6}{|l|}{ Gestação de alto risco } \\
\hline Não & $7.578(74,6)$ & $3.001(64,7)$ & $4.577(83,0)$ & 39,6 & $<0,001$ \\
\hline $\operatorname{Sim}$ & $2.577(25,4)$ & $1.638(35,3)$ & $939(17,0)$ & 63,5 & \\
\hline \multicolumn{6}{|l|}{ Obesidade } \\
\hline Não & $9.096(89,6)$ & $3.987(85,9)$ & $5.109(92,6)$ & 43,8 & $<0,001$ \\
\hline Sim & $1.059(10,4)$ & $652(14,1)$ & $407(7,4)$ & 61,5 & \\
\hline \multicolumn{6}{|l|}{ Macrossomia } \\
\hline Não & $9.741(95,9)$ & $4.410(95,1)$ & $5.331(96,6)$ & 45,2 & 0,04 \\
\hline $\operatorname{Sim}$ & $414(4,1)$ & $229(4,9)$ & $185(3,4)$ & 55,3 & \\
\hline \multicolumn{6}{|l|}{ Idade gestacional } \\
\hline Pré-termo & $1.050(10,3)$ & $504(10,9)$ & $546(9,9)$ & 48,0 & 0,22 \\
\hline Termo/Pós-termo & $9.105(89,7)$ & $4.135(89,1)$ & $4.970(90,1)$ & 45,4 & \\
\hline
\end{tabular}

UI: unidade intermediária; UTI: unidade de terapia intensiva.

Na Tabela 2, estratificamos as variáveis por fonte pagadora. Dentre as mulheres com financiamento público do parto, todas as características hospitalares testadas se associaram à cesárea eletiva, sendo esta mais frequente nos hospitais mistos, localizados fora da capital, de menor complexidade e menos de 1.500 partos/ano. Por outro lado, entre as mulheres com financiamento privado do parto, a complexidade hospitalar não foi associada à cesárea eletiva, sendo esta mais frequentemente utilizada nos hospitais privados do que nos mistos, em hospitais fora das capitais e com menor volume anual de partos.

Quanto às características socioeconômicas e obstétricas, todas as variáveis foram associadas à cesariana eletiva nas mulheres com financiamento público do parto, e nas mulheres com financiamento privado, morar com companheiro, gestação múltipla e macrossomia não estiveram associadas à cesariana eletiva.

De acordo com a análise multivariada dos partos com financiamento público (Tabela 3), observou-se que no modelo ajustado apenas para as características socioeconômicas e obstétricas, todas as características hospitalares analisadas apresentaram chance significativamente maior de cesárea eletiva. Contudo, quando foi realizado ajuste estatístico incluindo também as características hospitalares, 
Tabela 2

Características hospitalares, socioeconômicas e obstétricas das mulheres submetidas à cesariana eletiva e que tiveram cesariana intraparto/parto vaginal, estratificadas por fonte de financiamento do parto.

\begin{tabular}{|c|c|c|c|c|c|c|c|c|}
\hline \multirow[t]{3}{*}{ Características } & \multicolumn{4}{|c|}{ Fonte pagadora pública } & \multicolumn{4}{|c|}{ Fonte pagadora privada } \\
\hline & $\begin{array}{c}\text { Cesariana } \\
\text { eletiva }\end{array}$ & $\begin{array}{c}\text { Cesariana } \\
\text { intraparto } \\
\text { e parto } \\
\text { vaginal }\end{array}$ & $\begin{array}{c}\text { Prevalência } \\
\text { de cesariana } \\
\text { eletiva }\end{array}$ & $\begin{array}{l}\text { Valor } \\
\text { de } p\end{array}$ & $\begin{array}{l}\text { Cesariana } \\
\text { eletiva }\end{array}$ & $\begin{array}{c}\text { Cesariana } \\
\text { intraparto } \\
\text { e parto } \\
\text { vaginal }\end{array}$ & $\begin{array}{c}\text { Prevalência } \\
\text { de cesariana } \\
\text { eletiva }\end{array}$ & $\begin{array}{l}\text { Valor } \\
\text { de } p\end{array}$ \\
\hline & n (\%) & n (\%) & $\%$ & & n (\%) & n (\%) & $\%$ & \\
\hline \multicolumn{9}{|l|}{ Tipo de hospital } \\
\hline Público & $978(35,0)$ & $2.475(49,0)$ & 28,3 & $<0,001$ & - & - & & $<0,001$ \\
\hline Misto & $1,816(65,0)$ & $2.573(51,0)$ & 41,3 & & $534(29,0)$ & $204(43,6)$ & 72,3 & \\
\hline Privado & - & - & & & $1.311(71,0)$ & $264(56,4)$ & 83,2 & \\
\hline \multicolumn{9}{|l|}{ Localização do hospital } \\
\hline Capital & $736(26,3)$ & $1.914(37,9)$ & 27,7 & 0,002 & $585(31,7)$ & $194(41,4)$ & 75,1 & 0,003 \\
\hline Não capital & $2,058(73,7)$ & $3.135(62,1)$ & 39,6 & & $1.260(68,3)$ & $274(58,6)$ & 82,1 & \\
\hline \multicolumn{9}{|l|}{ Número de partos/ano } \\
\hline$<1,500$ & $994(35,6)$ & $1.169(23,1)$ & 45,9 & $<0,001$ & $745(40,4)$ & $137(29,3)$ & 84,4 & 0,04 \\
\hline $1,500-2,999$ & $827(29,6)$ & $1.229(24,3)$ & 40,2 & & $480(26,0)$ & $113(24,2)$ & 80,9 & \\
\hline$\geq 3,000$ & $973(34,8)$ & $2.651(52,5)$ & 26,8 & & $620(33,6)$ & $217(46,5)$ & 74,0 & \\
\hline \multicolumn{9}{|l|}{ Complexidade do hospital } \\
\hline Sem leitos de UI e UTI neonatal & $528(18,9)$ & $818(16,2)$ & 39,2 & $<0,001$ & $226(12,3)$ & $30(6,5)$ & 88,2 & 0,14 \\
\hline $\begin{array}{l}\text { Com leitos de UI neonatal, sem } \\
\text { UTI neonatal }\end{array}$ & $496(17,8)$ & $651(12,9)$ & 43,2 & & $81(4,4)$ & $50(10,7)$ & 61,8 & \\
\hline Tem leitos de UTI neonatal & $1.769(63,3)$ & $3.579(70,9)$ & 33,0 & & $1.537(83,3)$ & $387(82,8)$ & 79,8 & \\
\hline \multicolumn{9}{|l|}{ Idade (anos) } \\
\hline$\leq 19$ & $380(13,6)$ & $1.174(23,3)$ & 24,4 & $<0,001$ & $69(3,7)$ & $63(13,5)$ & 52,2 & 0,001 \\
\hline $20-34$ & $2.087(74,7)$ & $3.500(69,3)$ & 37,3 & & $1.413(76,6)$ & $341(73,1)$ & 80,5 & \\
\hline$\geq 35$ & $326(11,7)$ & $374(7,4)$ & 46,5 & & $364(19,7)$ & $63(13,4)$ & 85,2 & \\
\hline \multicolumn{9}{|l|}{ Anos de escolaridade } \\
\hline$\leq 7$ & $625(22,5)$ & $1.305(25,9)$ & 32,3 & 0,03 & $60(3,3)$ & $32(6,9)$ & 65,2 & 0,002 \\
\hline $8-10$ & $765(27,5)$ & $1.622(32,2)$ & 32,0 & & $165(9,0)$ & $84(18,2)$ & 66,2 & \\
\hline$\geq 11$ & $1.395(50,0)$ & $2.104(41,8)$ & 39,8 & & $1.611(87,7)$ & $346(74,9)$ & 82,3 & \\
\hline \multicolumn{9}{|l|}{ Situação conjugal } \\
\hline Não mora com o parceiro & $590(21,1)$ & $1.324(26,2)$ & 30,8 & 0,01 & $215(11,7)$ & $55(11,9)$ & 79,6 & 0,90 \\
\hline Mora com o parceiro & $2.203(78,9)$ & $3.721(73,8)$ & 37,2 & & $1.628(88,3)$ & $412(88,1)$ & 79,8 & \\
\hline \multicolumn{9}{|l|}{ Cesariana prévia } \\
\hline Não & $1.594(57,1)$ & $4.577(90,7)$ & 25,8 & $<0,001$ & $1.211(65,6)$ & $443(94,8)$ & 73,2 & $<0,001$ \\
\hline $\operatorname{Sim}$ & $1.199(42,9)$ & $471(9,3)$ & 71,8 & & $635(34,4)$ & $24(5,2)$ & 96,3 & \\
\hline \multicolumn{9}{|l|}{ Tipo de gestação } \\
\hline Única & $2.752(98,5)$ & $5.022(99,5)$ & 35,4 & 0,03 & $1.818(98,5)$ & $462(98,9)$ & 79,7 & 0,52 \\
\hline Múltipla & $42(1,5)$ & $27(0,5)$ & 60,8 & & $27(1,5)$ & $5(1,1)$ & 84,3 & \\
\hline \multicolumn{9}{|l|}{ Apresentação fetal } \\
\hline Cefálica & $2.571(92,0)$ & $4.969(98,4)$ & 34,1 & $<0,001$ & $1.760(95,4)$ & $458(97,9)$ & 79,3 & 0,02 \\
\hline Não cefálica & $222(8,0)$ & $80(1,6)$ & 73,5 & & $86(4,6)$ & $10(2,1)$ & 89,5 & \\
\hline \multicolumn{9}{|l|}{ Gestação de alto risco } \\
\hline Não & $1.667(59,7)$ & $4.196(83,1)$ & 28,4 & 0,04 & $1.334(72,3)$ & $380(81,4)$ & 77,8 & 0,03 \\
\hline Sim & $1.127(40,3)$ & $852(16,9)$ & 56,9 & & $511(27,7)$ & $87(18,6)$ & 85,4 & \\
\hline \multicolumn{9}{|l|}{ Obesidade } \\
\hline Não & $2.394(85,7)$ & $4.681(92,7)$ & 33,8 & $<0,001$ & $1.593(86,3)$ & $428(91,6)$ & 78,8 & 0,005 \\
\hline Sim & $400(14,3)$ & $368(7,3)$ & 52,0 & & $252(13,7)$ & $39(8,4)$ & 86,5 & \\
\hline
\end{tabular}

(continua) 
Tabela 2 (continuação)

\begin{tabular}{|c|c|c|c|c|c|c|c|c|}
\hline \multirow[t]{3}{*}{ Características } & \multicolumn{4}{|c|}{ Fonte pagadora pública } & \multicolumn{4}{|c|}{ Fonte pagadora privada } \\
\hline & $\begin{array}{c}\text { Cesariana } \\
\text { eletiva }\end{array}$ & $\begin{array}{c}\text { Cesariana } \\
\text { intraparto } \\
\text { e parto } \\
\text { vaginal }\end{array}$ & $\begin{array}{c}\text { Prevalência } \\
\text { de cesariana } \\
\text { eletiva }\end{array}$ & $\begin{array}{l}\text { Valor } \\
\text { de } p\end{array}$ & $\begin{array}{c}\text { Cesariana } \\
\text { eletiva }\end{array}$ & $\begin{array}{c}\text { Cesariana } \\
\text { intraparto } \\
\text { e parto } \\
\text { vaginal }\end{array}$ & $\begin{array}{c}\text { Prevalência } \\
\text { de cesariana } \\
\text { eletiva }\end{array}$ & $\begin{array}{l}\text { Valor } \\
\text { de } p\end{array}$ \\
\hline & n (\%) & n (\%) & $\%$ & & n (\%) & n (\%) & $\%$ & \\
\hline \multicolumn{9}{|l|}{ Macrossomia } \\
\hline Não & $2.631(94,2)$ & $4.879(96,6)$ & 35,0 & 0,02 & $1.779(96,4)$ & $452(96,7)$ & 79,7 & 0,58 \\
\hline Sim & $163(5,8)$ & $169(3,4)$ & 49,1 & & $66(3,6)$ & $15(3,3)$ & 81,4 & \\
\hline \multicolumn{9}{|l|}{ Idade gestacional } \\
\hline Pré-termo & $324(11,6)$ & $489(9,7)$ & 39,8 & 0,04 & $180(9,8)$ & $57(12,1)$ & 75,9 & 0,03 \\
\hline Termo/Pós-termo & $2.470(88,4)$ & $4.560(90,3)$ & 35,1 & & $1.665(90,2)$ & $410(87,9)$ & 80,2 & \\
\hline
\end{tabular}

UI: unidade intermediária; UTI: unidade de terapia intensiva.

Tabela 3

Razão de chance bruta e ajustada da cesariana eletiva segundo características hospitalares, socioeconômicas e obstétricas das mulheres com financiamento público do parto.

\begin{tabular}{|c|c|c|c|c|}
\hline Características & $\begin{array}{c}\text { Prevalência de } \\
\text { cesariana eletiva (\%) }\end{array}$ & OR bruta (IC95\%) & $\begin{array}{l}\text { OR ajustada } 1 \text { * } \\
\text { (IC95\%) }\end{array}$ & $\begin{array}{l}\text { OR ajustada } 2 \text { ** } \\
\text { (IC95\%) }\end{array}$ \\
\hline \multicolumn{5}{|l|}{ Tipo de hospital } \\
\hline Público & 28,3 & 1,00 & 1,00 & 1,00 \\
\hline Misto & 41,3 & $1,78(1,32-2,42)$ & $2,20(1,65-2,96)$ & $1,81(1,37-2,39)$ \\
\hline \multicolumn{5}{|l|}{ Localização do hospital } \\
\hline Capital & 27,7 & 1,00 & 1,00 & 1,00 \\
\hline Não capital & 39,6 & $1,70(1,23-2,38)$ & $2,04(1,53-2,75)$ & $1,35(0,98-1,84)$ \\
\hline \multicolumn{5}{|l|}{ Número de partos/ano } \\
\hline$<1.500$ & 45,9 & $2,31(1,67-3,21)$ & $2,62(1,84-3,75)$ & $2,11(1,37-3,26)$ \\
\hline $1.500-2.999$ & 40,2 & $1,83(1,34-2,51)$ & $1,92(1,32-2,81)$ & $1,45(1,04-2,02)$ \\
\hline$\geq 3.000$ & 26,8 & 1,00 & 1,00 & 1,00 \\
\hline \multicolumn{5}{|l|}{ Complexidade do hospital } \\
\hline Sem leitos de UI e UTI neonatal & 39,2 & $1,30(0,88-1,94)$ & $1,71(1,10-2,64)$ & $0,81(0,49-1,34)$ \\
\hline $\begin{array}{l}\text { Com leitos de UI neonatal, } \\
\text { sem UTI neonatal }\end{array}$ & 43,2 & $1,54(1,01-2,37)$ & $1,73(1,11-2,73)$ & $0,94(0,58-1,51)$ \\
\hline Tem leitos de UTI neonatal & 33,0 & 1,00 & 1,00 & 1,00 \\
\hline \multicolumn{5}{|l|}{ Idade (anos) } \\
\hline$\leq 19$ & 24,4 & $0,54(0,46-0,63)$ & $1,06(0,89-1,27)$ & $1,05(0,87-1,28)$ \\
\hline $20-34$ & 37,3 & 1,00 & 1,00 & 1,00 \\
\hline$\geq 35$ & 46,5 & $1,46(1,20-1,78)$ & $1,02(0,82-1,28)$ & $1,08(0,87-1,34)$ \\
\hline \multicolumn{5}{|l|}{ Anos de escolaridade } \\
\hline$\leq 7$ & 32,3 & $0,72(0,59-0,88)$ & $0,54(0,45-0,67)$ & $0,53(0,44-0,65)$ \\
\hline $8-10$ & 32,0 & $0,71(0,62-0,82)$ & $0,65(0,56-0,77)$ & $0,65(0,55-0,76)$ \\
\hline$\geq 11$ & 39,8 & 1,00 & 1,00 & 1,00 \\
\hline \multicolumn{5}{|l|}{ Situação conjugal } \\
\hline Não mora com o parceiro & 30,8 & $0,75(0,64-0,88)$ & $0,93(0,77-1,13)$ & $0,98(0,82-1,19)$ \\
\hline Mora com o parceiro & 37,2 & 1,00 & 1,00 & 1,00 \\
\hline
\end{tabular}

(continua) 
Tabela 3 (continuação)

\begin{tabular}{|c|c|c|c|c|}
\hline Características & $\begin{array}{c}\text { Prevalência de } \\
\text { cesariana eletiva }(\%)\end{array}$ & OR bruta (IC95\%) & $\begin{array}{l}\text { OR ajustada } 1 \text { * } \\
\text { (IC95\%) }\end{array}$ & $\begin{array}{l}\text { OR ajustada } 2 \text { ** } \\
\text { (IC95\%) }\end{array}$ \\
\hline \multicolumn{5}{|l|}{ Cesariana prévia } \\
\hline Não & 25,8 & 1,00 & 1,00 & 1,00 \\
\hline Sim & 71,8 & $7,30(5,66-9,43)$ & $8,48(6,40-11,24)$ & $8,91(6,76-11,74)$ \\
\hline \multicolumn{5}{|l|}{ Tipo de gestação } \\
\hline Única & 35,4 & 1,00 & 1,00 & 1,00 \\
\hline Múltipla & 60,8 & $2,79(1,36-5,76)$ & $1,52(0,64-3,62)$ & $1,47(0,60-3,58)$ \\
\hline \multicolumn{5}{|l|}{ Apresentação fetal } \\
\hline Cefálica & 34,1 & 1,00 & 1,00 & 1,00 \\
\hline Não cefálica & 73,5 & $5,38(3,99-7,26)$ & $6,89(4,83-9,86)$ & $7,17(5,18-9,93)$ \\
\hline \multicolumn{5}{|l|}{ Gestação de alto risco } \\
\hline Não & 28,4 & 1,00 & 1,00 & 1,00 \\
\hline Sim & 56,9 & $3,32(2,82-3,93)$ & $3,40(2,87-4,03)$ & $3,69(3,11-4,38)$ \\
\hline \multicolumn{5}{|l|}{ Obesidade } \\
\hline Não & 33,8 & 1,00 & 1,00 & 1,00 \\
\hline Sim & 52,0 & $2,12(1,79-2,52)$ & $1,38(1,13-1,68)$ & $1,33(1,09-1,63)$ \\
\hline \multicolumn{5}{|l|}{ Macrossomia } \\
\hline Não & 35,0 & 1,00 & 1,00 & 1,00 \\
\hline Sim & 49,1 & $1,77(1,33-2,38)$ & $1,83(1,28-2,62)$ & $1,97(1,36-2,86)$ \\
\hline \multicolumn{5}{|l|}{ Idade gestacional } \\
\hline Pré-termo & 39,8 & $1,22(1,01-1,47)$ & $0,97(0,79-1,21)$ & $1,10(0,86-1,42)$ \\
\hline Termo/Pós-termo & 35,1 & 1,00 & 1,00 & 1,00 \\
\hline
\end{tabular}

IC95\%: intervalo de 95\% de confiança; OR: odds ratio; UI: unidade intermediária; UTI: unidade de terapia intensiva.

* Ajustada por idade, escolaridade, situação conjugal, cesariana prévia, tipo de gestação, apresentação fetal, gestação de alto risco, obesidade, macrossomia, idade gestacional;

** Ajustada por variáveis do primeiro ajuste e tipo de hospital, localização do hospital, número de partos/ano e complexidade do hospital.

apenas o tipo de hospital misto e o menor volume anual de partos mantiveram associação significativa com cesárea eletiva. No modelo final, a chance de cesárea eletiva foi maior nas mulheres que foram atendidas nos hospitais mistos ( $\mathrm{OR}=1,81$; IC95\%: 1,37-2,39), naqueles com menos de 1.500 (OR = 2,11; IC95\%: 1,37-3,26) e entre 1.500-2.999 (OR = 1,45; IC95\%: 1,04-2,02) partos/ano, naquelas com cesárea prévia $(\mathrm{OR}=8,91$; IC95\%: 6,76-11,74), apresentação não cefálica (OR = 7,17; IC95\%: 5,189,93), gestações de alto risco (OR = 3,69; IC95\%: 3,11-4,38), obesas (OR = 1,33; IC95\%: 1,09-1,63) e com macrossomia (OR = 1,97; IC95\%: 1,36-2,86). Por outro lado, a chance de cesárea eletiva foi menor nas mulheres com menor escolaridade (OR = 0,53; IC95\%: 0,44-0,65, quando escolaridade $\leq 7$ anos; $\mathrm{OR}=0,65$; IC95\%: 0,55-0,76, quando escolaridade 8-10 anos).

A análise da Tabela 4 apontou maiores chances de realização de cesarianas eletivas nos hospitais mistos em geral, a magnitude da associação aumenta nos hospitais localizados em não capitais com mais de 3.000 partos (OR = 3,45; IC95\%: 1,68-7,08) e atinge seu maior valor nos hospitais mistos de não capitais com menos de 3.000 partos, apresentando 4 vezes mais chances de ser realizada uma cesariana eletiva (OR = 4,08; IC95\%: 2,61-6,37). Em contrapartida, não observou-se associação entre cesariana eletiva e os hospitais públicos localizados em não capitais do Sudeste.

\section{Discussão}

A assistência obstétrica no Brasil é marcada por sua elevada prevalência de cesarianas. Apesar do grande número de cesarianas eletivas realizadas na saúde suplementar ser um importante contribuinte para esse cenário 8 , ainda nos deparamos com alta prevalência dessa cirurgia mesmo entre as 


\section{Tabela 4}

Prevalência de cesariana eletiva e razões de chance bruta e ajustada segundo combinações de características hospitalares.

\begin{tabular}{lccc}
\hline Combinações & Cesariana eletiva & OR bruta (IC95\%) & $\begin{array}{c}\text { OR ajustada } \\
\text { (IC95\%) * }\end{array}$ \\
\hline Capital, SUS, $\geq 3.000(\mathrm{n}=1.203)$ & $317(26,4)$ & Referência & Referência \\
Capital, SUS, $<3.000(\mathrm{n}=370)$ & $154(41,6)$ & $1,99(0,74-5,33)$ & $2,26(1,08-4,73)$ \\
Capital, Misto, $\geq 3.000(\mathrm{n}=1.227)$ & $327(26,7)$ & $1,01(0,66-1,55)$ & $1,68(1,07-7,08)$ \\
Não capital, Misto, $\geq 3.000(\mathrm{n}=521)$ & $228(43,8)$ & $2,17(1,62-2,93)$ & $3,45(1,68-7,08)$ \\
Não capital, Misto, $<3.000(\mathrm{n}=3.379)$ & $1.795(53,1)$ & $3,16(2,36-4,24)$ & $4,08(2,61-6,37)$ \\
Não capital, SUS, $\geq 3.000(\mathrm{n}=913)$ & $233(25,5)$ & $0,95(0,72-1,27)$ & $1,10(0,63-1,91)$ \\
Não capital, SUS, $<3.000(\mathrm{n}=966)$ & $273(28,3)$ & $1,10(0,73-1,64)$ & $1,25(0,71-2,19)$ \\
Privado $(\mathrm{n}=1.575)$ & $1.311(83,2)$ & $13,87(8,68-22,14)$ & $14,72(8,32-26,05)$
\end{tabular}

IC95\%: intervalo de 95\% de confiança; OR: odds ratio; SUS: Sistema Único de Saúde.

* Ajustada por idade, escolaridade, situação conjugal, cesariana prévia, tipo de gestação, apresentação fetal, gestação de alto risco, obesidade, macrossomia, idade gestacional, complexidade do hospital.

usuárias do SUS. Observamos, na presente análise, que a prevalência de cesariana eletiva na Região Sudeste foi de $45,7 \%$, atingindo $83,2 \%$ nos hospitais privados.

Segundo informações disponíveis no SINASC (http://tabnet.datasus.gov.br/cgi/tabcgi.exe?si nasc/cnv/nvuf.def, acessado em 16/Mai/2019), em 2017, o percentual de cesarianas anteparto na Região Sudeste foi de 35,5\%, inferior ao encontrado em nosso estudo, o que poderia ser atribuído tanto a uma mudança na forma de assistência ao parto no país, como a diferenças na definição de cesárea anteparto. Esse percentual é, entretanto, mais que o dobro daquele encontrado nas regiões Norte $(15,2 \%)$ e Nordeste $(17,7 \%)$ e ligeiramente inferior ao da Região Sul (38,8\%). Quando analisamos apenas os partos com financiamento público, encontramos $26,4 \%$ de mulheres que foram submetidas à cesárea eletiva na Região Sudeste, número também inferior ao encontrado na presente análise (35,6\% - dado não mostrado). Uma vez mais, esse percentual é superior àqueles encontrados nas regiões Norte (11,9\%) e Nordeste (12,9\%) e inferior ao da Região Sul (32,6\%). Esses dados podem apontar para uma maior dificuldade de acesso aos serviços de saúde nas regiões Norte e Nordeste, ainda que os números encontrados nas regiões Sul e Sudeste denotem um uso excessivo da cesárea eletiva, mesmo no sistema público. No presente estudo, a prevalência de cesarianas eletivas foi de 28,3\% nos hospitais estritamente públicos, sendo quase três vezes maior do que na França $(10,9 \%) 16$ e quatro vezes maior do que na Holanda (6,9\%) 17.

Os resultados da nossa pesquisa apontam a importância das características hospitalares para a ocorrência da cesárea eletiva na Região Sudeste. Conforme já observado em inúmeros outros estudos $8,12,18,19$, o financiamento privado aumenta sobremaneira a chance da cesárea eletiva. Por outro lado, observamos também que, além do financiamento, algumas características hospitalares no setor público estão associadas com maior chance de cesariana eletiva, particularmente o volume de partos por ano e a localização do hospital em cidades não capitais.

As características hospitalares como localização geográfica, infraestrutura, volume de partos, recursos humanos, entre outras, têm se tornado alvo de estudos 10,18,20,21,22,23, tanto para avaliação da adequação de recursos quanto para avaliação da influência destas características sobre desfechos de interesse, tais como mortalidade neonatal e cesariana. Algumas investigações realizadas a fim de elucidar os fatores relacionados às altas prevalências de cesariana demonstraram que, após o ajuste para as variáveis clínicas e sociodemográficas, estas prevalências permaneciam altas e variavam sensivelmente entre os hospitais 13,18,24, ou seja, a prevalência de cesárea nem sempre estava relacionada ao perfil de risco da população atendida. Isso indica que o local de atendimento das gestantes também é um importante influenciador na via de parto, pois acredita-se que fatores como a variação de protocolos entre as maternidades, a adesão dos profissionais aos protocolos já estabelecidos, as diferentes 
rotinas de atendimento, a infraestrutura das maternidades, assim como a presença ou não de equipe multidisciplinar podem estar diretamente relacionados com a via de parto 12,19,25,26.

$\mathrm{Na}$ análise geral, os hospitais mistos apresentaram uma prevalência de cesariana eletiva de 45,8\%, já nos hospitais públicos esta prevalência foi de 28,3\%. Em princípio esse achado poderia ser atribuído à parcela de partos financiados pelo setor privado. Entretanto, após a análise estratificada por fonte pagadora pública, observa-se ainda uma prevalência de $41,3 \%$, e uma chance $80 \%$ maior de cesariana eletiva nesses hospitais, mesmo nos atendimentos financiados pelo SUS. Um estudo que analisou a prevalência de cesarianas nos hospitais brasileiros, selecionando hospitais públicos e mistos, também observou maior proporção de cesarianas nos hospitais com financiamento misto 27 . As razões para tais achados ainda não estão elucidadas, necessitando de trabalhos específicos para este fim. Entretanto, pode-se levantar a hipótese de que o tipo de assistência prestada nesses hospitais seja semelhante para todos os partos, independentemente da fonte de financiamento. Isso implicaria haver uma forma de "contaminação" dos índices habitualmente observados nos hospitais com financiamento público exclusivo, por conta da coexistência com o financiamento privado. Essa "contaminação" aconteceria pela replicação da forma de organização dos hospitais privados, com agendamento prévio de cesárea, tanto por desejo materno, como por comodidade médica.

A complexidade do hospital não esteve associada à realização de cesárea eletiva no modelo final, diferentemente do encontrado em alguns estudos realizados em outros países 19,21. Padua et al. 27 também não verificaram associação da complexidade do hospital com cesariana no Brasil. É interessante notar, entretanto, que quando ajustada somente pelas características socioeconômicas e hospitalares, a chance de cesariana foi maior nos hospitais com estrutura mais precária, quando esperava-se encontrar mais cesarianas eletivas nos hospitais com UTI neonatal, geralmente aptos a receber gestações mais complicadas.

Os hospitais localizados fora das capitais não apresentaram, na análise ajustada para todas as variáveis, associação significativamente estatística para a realização de cesariana eletiva. No entanto, após a realização de ajuste apenas para as características clínicas e socioeconômicas, a chance de cesariana eletiva foi duas vezes maior, demonstrando que em não capitais outras características hospitalares possivelmente atuam para o incremento na prevalência de cesariana eletiva. Isso foi perceptível na análise apresentada na Tabela 4, uma vez que, entre os hospitais localizados em não capitais, os mistos apresentaram maior chance de cesariana eletiva independentemente do volume de partos/ano, quando comparados com os hospitais do SUS. Assim, podemos afirmar que só o fato de um hospital ser misto já potencializa a chance de cesariana eletiva, independentemente das outras características hospitalares.

Ressalta-se que os hospitais com mais de 3.000 partos/ano são os que apresentam a menor prevalência de cesarianas (35,7\%), quando comparados com os hospitais com 1.500 a 2.999 partos/ano $(49,3 \%)$ e com aqueles com < 1.500 partos/ano (57,1\%). Essa relação se mantém na estratificação quanto ao tipo de financiamento do parto, porém, observa-se que a diferença é maior nos partos pagos pelo SUS, em que há chances duas vezes superiores de cesárea eletiva nos hospitais com < 1.500 partos/ ano. O volume de partos do hospital tem especial relevância nos hospitais públicos das capitais, mais do que dobrando a chance de cesárea eletiva. A associação do volume anual de partos com cesariana 18,24 ou cesariana de repetição 19 não foi identificada em estudos prévios realizados em países desenvolvidos. Um estudo realizado na Coreia do Sul, no entanto, verificou que a ocorrência de cesarianas era maior em hospitais com volume abaixo da mediana 28. Os autores atribuíram essa associação à necessidade de hospitais com menor volume de partos manterem seus leitos ocupados com pacientes que terão recuperação mais demorada e com maior reembolso financeiro ao hospital 28. É provável que em hospitais com reduzido número de partos/ano, a via de parto seja influenciada por questões organizacionais, como menor número de médicos por equipe, tomadas de decisão centradas em apenas um profissional, agendamento de cesarianas para o melhor fluxo do serviço, entre outras.

Os dados expostos nesta análise e as hipóteses aventadas trazem a discussão para o campo da organização, gerenciamento e financiamento do sistema de saúde. O próprio Ministério da Saúde reconhece a carência nesse setor, tendo publicado em 30 de dezembro de 2010 a Portaria no 4.27929 , estabelecendo diretrizes para a organização da Rede de Atenção à Saúde no âmbito do SUS. O texto da Portaria reconhece a fragmentação do sistema e a pulverização dos mesmos serviços em nível municipal como um problema a ser enfrentado. Nesse contexto, a organização da atenção à saúde, 
por meio de redes regionalizadas, permitiria uma melhor alocação dos recursos, priorizando hospitais maiores, com melhor infraestrutura para atender a população, a fim de reduzir o número de hospitais pequenos, que oneram e fragmentam o sistema. Hospitais com maior volume de partos, além de menores porcentuais de cesarianas como evidenciado no presente estudo, também podem impactar outros desfechos relevantes, como menor mortalidade neonatal 22 e menor morbidade materna 30.

De todo o exposto, torna-se clara a necessidade de o país reestruturar sua rede hospitalar de assistência obstétrica. Tal reestruturação passa necessariamente pela reorganização da assistência em uma rede regionalizada, centrada em hospitais maiores, com maior volume de partos e, preferencialmente, estritamente públicos 31 . Essa rede deve ter suficiência de leitos para gestação de alto risco, de UI e UTI neonatal, e também de UTI obstétrica. As maternidades qualificadas como referência para alto risco materno devem preferencialmente ter UTI obstétrica e, quando não tiverem, devem ter acordo com hospital que conte com leito de UTI para evitar demoras no atendimento ${ }^{31}$. Além disso, deverá contar com coordenação adequada, recursos humanos qualificados e financiamento suficiente. Um segundo ponto é a organização de um fluxo eficiente de informação no sistema, de forma a disseminar o consenso sobre as boas práticas ligadas ao parto.

Um dos pontos fortes deste artigo é que não identificamos estudos prévios no Brasil que tenham investigado a associação de características hospitalares com a chance de cesariana, cujas análises tenham incluído o volume anual de partos e a localização geográfica em capitais ou não dos hospitais. Outro ponto forte é que a amostra do Nascer no Brasil foi realizada de forma a ter representação para todas as regiões do país. Por outro lado, não foram incluídos os estabelecimentos com menos de 500 partos/ano, que são responsáveis por 20\% dos nascimentos no Brasil. Entretanto, como verificamos que quanto menor o hospital, maior a prevalência de cesariana eletiva, a não inclusão desses hospitais estaria possivelmente subestimando as razões de chance para cesariana eletiva dos hospitais com menos de 1.500 partos/ano. Outra limitação é a definição de cesariana eletiva neste estudo, que considerou mulheres que entraram em trabalho de parto apenas aquelas que atingiram dilatação maior ou igual a $4 \mathrm{~cm}$, possivelmente englobando na variável de cesariana eletiva algumas parturientes na fase latente do trabalho de parto. Além disso, não avaliamos a indicação de cesárea e se a mesma foi realizada por desejo materno.

Apesar do estudo não ter sido desenhado para o propósito deste artigo, foi possível trazer algumas questões importantes sobre o tipo de assistência prestada nos hospitais da Região Sudeste. Por ser a região mais industrializada e com maior poder econômico do país, é a que conta com os melhores indicadores de assistência, entretanto, entendemos que ainda há muitos desafios a serem transpostos. 


\section{Colaboradores}

L. Zaiden e M. Nakamura-Pereira contribuíram com a concepção, análise dos dados, redação do artigo e aprovação da versão final. M. A. M. Gomes contribuiu com a concepção, revisão crítica do artigo e aprovação da versão final. A. P. Esteves-Pereira e M. C. Leal contribuíram com a aquisição dos dados, revisão crítica do artigo e aprovação da versão final.

\section{Informações adicionais}

ORCID: Laura Zaiden (0000-0001-5074-708X); Marcos Nakamura-Pereira (0000-0002-42310205); Maria Auxiliadora Mendes Gomes (00000001-5908-1763); Ana Paula Esteves-Pereira (0000-0002-0236-2043); Maria do Carmo Leal (0000-0002-3047-515X).

\section{Referências}

1. Betran AP, Torloni MR, Zhang J, Ye J, Mikolajczyk R, Deneux-Tharaux C, et al. What is the optimal rate of caesarean section at population level? A systematic review of ecologic studies. Reprod Health 2015; 12:57.

2. Ye J, Zhang J, Mikolajczyk R, Torloni MR, Gulmezoglu AM, Betran AP. Association between rates of caesarean section and maternal and neonatal mortality in the 21st century: a worldwide population-based ecological study with longitudinal data. BJOG 2016; 123:745-53.

3. Esteves-Pereira AP, Deneux-Tharaux C, Nakamura-Pereira M, Saucedo M, BouvierColle MH, Leal MC. Caesarean delivery and postpartum maternal mortality: a populationbased case control study in Brazil. PLoS One 2016; 11:e0153396.

4. Liu S, Liston RM, Joseph KS, Heaman M, Sauve R, Kramer MS, et al. Maternal mortality and severe morbidity associated with low-risk planned cesarean delivery versus planned vaginal delivery at term. CMAJ 2007; 176:455-60.

5. Villar J, Carroli G, Zavaleta N, Donner A, Wojdyla D, Faundes A, et al. Maternal and neonatal individual risks and benefits associated with caesarean delivery: multicentre prospective study. BMJ 2007; 335:1025.

\section{Agradecimentos}

O estudo Nascer no Brasil foi financiado por: Conselho Nacional de Desenvolvimento Científico e Tecnológico (CNPq); Departamento de Ciência e Tecnologia, Secretaria de Ciência, Tecnologia de Insumos Estratégicos, Ministério da Saúde; Escola Nacional de Saúde Pública Sergio Arouca, Fundação Oswaldo Cruz (Projeto INOVA); e Fundação de Amparo à Pesquisa do Estado do Rio de Janeiro (Faperj).
6. Xie RH, Gaudet L, Krewski D, Graham ID, Walker MC, Wen SW. Higher cesarean delivery rates are associated with higher infant mortality rates in industrialized countries. Birth 2015; 42:62-9.

7. Ramires de Jesus G, Ramires de Jesus N, Peixoto-Filho FM, Lobato G. Caesarean rates in Brazil: what is involved? BJOG 2015; 122:606-9.

8. Nakamura-Pereira M, do Carmo Leal M, Esteves-Pereira AP, Domingues RM, Torres JA, Dias MA, et al. Use of Robson classification to assess cesarean section rate in Brazil: the role of source of payment for childbirth. Reprod Health 2016; 13 Suppl 3:128.

9. Machado JP, Martins M, Leite IC. O mix público-privado e os arranjos de financiamento hospitalar no Brasil. Saúde Debate 2015; 39:39-50.

10. Nakamura-Pereira M, Esteves-Pereira AP, Gama SGN, Leal M. Elective repeat cesarean delivery in women eligible for trial of labor in Brazil. Int J Gynaecol Obstet 2018; 143:351-9.

11. Nippita TA, Lee YY, Patterson JA, Ford JB, Morris JM, Nicholl MC, et al. Variation in hospital caesarean section rates and obstetric outcomes among nulliparae at term: a populationbased cohort study. BJOG 2015; 122:702-11. 
12. Schemann K, Patterson JA, Nippita TA, Ford JB, Roberts CL. Variation in hospital caesarean section rates for women with at least one previous caesarean section: a population based cohort study. BMC Pregnancy Childbirth $2015 ; 15: 179$.

13. Caceres IA, Arcaya M, Declercq E, Belanoff CM, Janakiraman V, Cohen B, et al. Hospital differences in cesarean deliveries in Massachusetts (US) 2004-2006: the case against casemix artifact. PLoS One 2013; 8:e57817.

14. do Carmo Leal M, da Silva AA, Dias MA, da Gama SG, Rattner D, Moreira ME, et al. Birth in Brazil: national survey into labour and birth. Reprod Health 2012; 9:15.

15. Vasconcellos MT, Silva PL, Pereira APE, Schilithz AOC, Souza Junior PRB, Szwarcwald CL. Desenho da amostra Nascer no Brasil: Pesquisa Nacional sobre Parto e Nascimento. Cad Saúde Pública 2014; 30 Suppl 1:S49-58.

16. Coulm B, Blondel B, Alexander S, Boulvain M, Le Ray C. Potential avoidability of planned cesarean sections in a French national database. Acta Obstet Gynecol Scand 2014; 93:905-12.

17. Zhang J, Geerts C, Hukkelhoven C, Offerhaus $\mathrm{P}$, Zwart J, de Jonge A. Caesarean section rates in subgroups of women and perinatal outcomes. BJOG 2016; 123:754-61.

18. Sebastião YV, Womack L, Vamos CA, Louis JM, Olaoye F, Caragan T, et al. Hospital variation in cesarean delivery rates: contribution of individual and hospital factors in Florida. Am J Obstet Gynecol 2016; 214:123.e1-123.e18.

19. Bartolo S, Goffinet F, Blondel B, Deneux-Tharaux C. Why women with previous caesarean and eligible for a trial of labour have an elective repeat caesarean delivery? A national study in France. BJOG 2016; 123:1664-73.

20. Bittencourt SDA, Reis LGC, Ramos MM, Rattner D, Rodrigues PL, Neves DCO, et al. Estrutura das maternidades: aspectos relevantes para a qualidade da atenção ao parto e nascimento. Cad Saúde Pública 2014; 30 Suppl $1:$ S208-19.

21. Little SE, Orav EJ, Robinson JN, Caughey AB, Jha AK. The relationship between variations in cesarean delivery and regional health care use in the United States. Am J Obstet Gynecol 2016; 214:735.e1-8.
22. Moster D, Lie RT, Markestad T. Neonatal mortality rates in communities with small maternity units compared with those having larger maternity units. BJOG 2001; 108:904-9.

23. Bittencourt SD, Domingues RM, Reis LG, Ramos MM, Leal MD. Adequacy of public maternal care services in Brazil. Reprod Health 2016; 13 Suppl 3:120.

24. Bragg F, Cromwell DA, Edozien LC, GurolUrganci I, Mahmood TA, Templeton A, et al. Variation in rates of caesarean section among English NHS trusts after accounting for maternal and clinical risk: cross sectional study. BMJ 2010; 341:c5065.

25. Vogt SE, Diniz SG, Tavares CM, Santos NCP, Schneck CA, Zorzam B, et al. Características da assistência ao trabalho de parto e parto em três modelos de atenção no SUS, no Município de Belo Horizonte, Minas Gerais, Brasil. Cad Saúde Pública 2011; 27:1789-800.

26. Miller S, Abalos E, Chamillard M, Ciapponi A, Colaci D, Comandé D, et al. Beyond too little, too late and too much, too soon: a pathway towards evidence-based, respectful maternity care worldwide. Lancet 2016; 388:2176-92.

27. Padua KS, Osis MJ, Faundes A, Barbosa AH, Moraes Filho OB. Factors associated with cesarean sections in Brazilian hospitals. Rev Saúde Pública 2010; 44:70-9.

28. Han KT, Kim SJ, Ju YJ, Choi JW, Park EC. Do hospital characteristics influence cesarean delivery? Analysis of National Health Insurance claim data. Eur J Public Health 2017; 27:801-7.

29. Ministério da Saúde. Portaria no 4.279, de 30 de dezembro de 2010. Estabelece diretrizes para organização da Rede de Atenção no âmbito do Sistema Único de Saúde (SUS). Diário Oficial da União 2010; 31 dez.

30. Aubrey-Bassler FK, Cullen RM, Simms A, Asghari S, Crane J, Wang PP, et al. Populationbased cohort study of hospital delivery volume, geographic accessibility, and obstetric outcomes. Int J Gynecol Obstet 2019; 146:95-102.

31. Pacagnella RC, Nakamura-Pereira M, GomesSponholz F, Aguiar RALP, Guerra GVQL, Diniz CSG, et al. Maternal mortality in Brazil: proposals and strategies for its reduction. Rev Bras Ginecol Obstet 2018; 40:501-6. 


\section{Abstract}

This article aims to assess the influence of hospital characteristics on the odds of performing an elective cesarean in the Southeast region of Brazil. Data were obtained from the Birth in Brazil study, conducted from February 2011 to October 2012. The current analysis includes the sample from Southeast Brazil, with 10,155 women. The group of women that underwent elective cesareans was compared to the women who went into labor or underwent labor induction, regardless they had intrapartum cesarean or vaginal delivery. Except for gestational age, all the obstetric characteristics analyzed were associated with elective cesarean. In this group, $60.5 \%$ had no prior cesarean and $64.7 \%$ had low-risk gestations. Among the births with public financing, there were higher odds of elective cesareans in women treated at hospitals with < 1,500 births/year $(\mathrm{OR}=2.11$; 95\%CI: 1.37-3.26) and 1,500-2,999 births/year $(\mathrm{OR}=1.45$; 95\% CI: 1.04-2.02) and in mixed hospitals $(O R=1.81$; 95\%CI: 1.37-2.39). In the mixed hospitals, the association was stronger when located in non-capital cities with $>3,000$ births/year $(O R=3.45 ; 95 \% \mathrm{CI}$ : 1.68-7.08), reaching the highest level in hospitals in non-capital cities with $<3,000 \mathrm{births} /$ year $(\mathrm{OR}=$ 4.08; 95\%CI: 2.61-6.37). Meanwhile, no association was seen between elective cesarean and public hospitals located in non-capital cities of the Southeast region. Prevalence rates of elective cesareans in public hospitals in Southeast Brazil are high when compared to other countries, and they are heavily influenced by hospital characteristics.

Cesarean Section; Natural Childbirth; Unified Health System; Public Hospitals; Induced Labor

\section{Resumen}

El objetivo de este artículo es evaluar la influencia de las características hospitalarias sobre la oportunidad de realización de cesáreas electivas en la región sudeste de Brasil. Se utilizan datos de la investigación Nacer en Brasil, realizada entre febrero de 2011 y octubre de 2012. El presente análisis incluye la muestra de la región sudeste, comprendiendo a 10.155 mujeres. El grupo de mujeres sometidas a una cesárea electiva se comparó con el de mujeres que entraron en trabajo de parto o fueron sometidas a la inducción del parto, independientemente si tuvieron cesárea intraparto o parto vaginal. Con excepción de la edad gestacional, todas las características obstétricas analizadas se mostraron asociadas con la cesárea electiva. En ese grupo un 60,5\% no tuvieron una cesárea previa y un 64,7\% tenían gestaciones de riesgo bajo. Entre los partos con financiación pública se observó una mayor oportunidad de cesárea electiva en las mujeres que fueron atendidas en los hospitales con < $1.500(O R=2,11$; IC95\%: 1,37-3,26) y entre 1.500-2.999 partos/año $(O R=1,45$; IC95\%: 1,04-2,02) y en los hospitales mixtos $(O R=1,81$; IC95\%: 1,37-2,39). En los hospitales mixtos, la magnitud de la asociación es mayor cuando están localizados fuera de la capital (OR = 3,45; IC95\%: 1,68-7,08), en los con volumen $>3.000$ partos/año, $y$ alcanza su mayor valor en los hospitales fuera de las capitales con volumen $<3.000$ partos/año $(\mathrm{OR}=4,08$; IC95\%: 2,61-6,37). Como contrapartida, no se observó asociación entre cesárea electiva y los hospitales públicos localizados fuera de las capitales del sudeste. Las prevalencias de cesárea electiva en los hospitales públicos de la región sudeste son altas, cuando se comparan con las de otros países, $y$ sufren una importante influencia de las características hospitalarias.

Cesárea; Parto Normal; Sistema Único de Salud; Hospitales Públicos; Trabajo de Parto Inducido
Recebido em 14/Nov/2018

Versão final reapresentada em 18/Jun/2019

Aprovado em 08/Jul/2019 